\title{
Long-Term Sensitization Training Produces Spike Narrowing in Aplysia Sensory Neurons
}

\author{
Evangelos G. Antzoulatos and John H. Byrne \\ Department of Neurobiology and Anatomy, W. M. Keck Center for the Neurobiology of Learning and Memory, The University of Texas Medical School at \\ Houston, Houston, Texas 77030
}

\begin{abstract}
Both short- and long-term sensitization of withdrawal reflexes of Aplysia are attributable at least in part to facilitation of the sensorimotor synapse. Previously, short-term synaptic facilitation has been associated with spike broadening and no change in temporal dynamics of burst transmission. In the present study, we examined whether long-term sensitization (LTS) is also associated with spike broadening and whether long-term synaptic facilitation is accompanied by changes in temporal dynamics. The results indicate that the temporal dynamics of the sensorimotor synapse are preserved after long-term facilitation. However, in contrast to short-term sensitization, LTS was accompanied by spike narrowing. The spike narrowing was observed both in centrally triggered spikes in isolated ganglia and in peripherally triggered spikes in reduced tail preparations. In addition, in reduced tail preparations, fewer spike failures in the afferent discharge of sensory neurons occurred in response to tail stimulation after ipsilateral LTS. Collectively, the results reveal that long-term sensitization affects the spike waveform of sensory neurons and enhances the sensory neuron responses to peripheral stimuli, but does not modify the synaptic dynamics of homosynaptic depression.
\end{abstract}

Key words: plasticity; spike waveform; spike width; spike broadening; synaptic facilitation; synaptic depression

\section{Introduction}

Sensitization of withdrawal reflexes in the marine mollusc Aplysia has been used extensively as a model system of learning and memory. Sensitization is a simple form of nonassociative learning in which responses elicited by weak test stimuli are augmented by training with strong, usually noxious stimuli (Carew et al., 1971; Pinsker et al., 1973; Scholz and Byrne, 1987). Both the short- and long-term memory for sensitization have been associated with changes in the properties of sensory neurons and their glutamatergic synapses (Antzoulatos and Byrne, 2004). Shortterm sensitization is partly attributed to facilitation of transmitter release from the presynaptic sensory neurons (Castellucci and Kandel, 1976; Byrne and Kandel, 1996). The increased transmitter release is associated with the well characterized broadening of sensory neuron spikes (Klein and Kandel, 1980; Klein et al., 1982; Baxter and Byrne, 1989; Baxter et al., 1999) and with a spikeduration-independent process, which presumably involves the mobilization of transmitter for release (Angers et al., 2002).

Long-term sensitization is associated with long-term facilitation of sensorimotor synapses (Frost et al., 1985; Cleary et al.,

Received May 26, 2006; revised Dec. 8, 2006; accepted Dec. 11, 2006.

This work was supported by National Institutes of Health Grant R01 NS19895. We thank Jennifer DeLeon and Marcy Wainwright for their assistance with behavioral training, and L. Cleary, D. Fioravante, and G. Phares for their comments on a previous draft of this manuscript.

Correspondence should be addressed to John H. Byrne, Department of Neurobiology and Anatomy, W. M. Keck Center for the Neurobiology of Learning and Memory, The University of Texas Medical School at Houston, 6431 Fannin Street, 7.046, Houston, TX 77030. E-mail: John.H.Byrne@uth.tmc.edu.

E. G. Antzoulatos' present address: The Picower Institute for Learning and Memory, Massachusetts Institute of Technology, Cambridge, MA 02139.

DOI:10.1523/JNEUROSCI.4025-06.2007

Copyright $\odot 2007$ Society for Neuroscience $\quad$ 0270-6474/07/270676-08\$15.00/0
1998; Wainwright et al., 2004) and in vitro analogues indicate that long-term facilitation, like short-term facilitation, is mediated by increases in presynaptic transmitter release (Dale et al., 1988). If so, one might expect that long-term facilitation, like short-term facilitation, is associated with spike broadening. Surprisingly, this hypothesis has never been examined. A second hypothesis, which is an extension of the first, is that long-term facilitation should be associated with an enhancement of the rate of homosynaptic depression during repetitive stimulation of sensory neurons. The enhanced fractional release produced by the broadened spike would enhance the depletion of the pool of available transmitter so that less would be available for release by subsequent spikes (Gingrich and Byrne, 1985). In addition, increased levels of transmitter release would augment the desensitization of glutamate receptors and, consequently, the rate of synaptic depression at high frequencies of stimulation (Antzoulatos et al., 2003).

In the present study, we tested these two hypotheses. We found that long-term sensitization training affects the spike waveform in the opposite direction to short-term sensitization (i.e., decreases the spike width), but does not change the dynamics of homosynaptic depression. The surprising finding that longterm sensitization training produces spike narrowing indicates that changes in spike waveform do not contribute directly to long-term synaptic facilitation. Long-term sensitization training also enhanced the peripherally evoked responses of sensory neurons to test stimuli by reducing the probability of spike failures during high-frequency discharges.

\section{Materials and Methods}

General. Aplysia californica (100-300 g) were obtained from Alacrity Marine Biological Specimens (Redondo Beach, CA) and Marinus (Long 
Beach, CA). Animals were individually caged in aquaria containing artificial seawater (ASW; Instant Ocean, Mentor, $\mathrm{OH}$ ) at $\sim 15^{\circ} \mathrm{C}$ with a $12 \mathrm{~h}$ light/dark cycle, and were fed dried seaweed three times per week. Animal dissections and in vitro experiments were conducted as described below in the following media (Trudeau and Castellucci, 1992). Normal ASW contained the following (in mM): $460 \mathrm{NaCl}, 10 \mathrm{KCl}, 11 \mathrm{CaCl}_{2}, 30 \mathrm{MgCl}_{2}$, $25 \mathrm{MgSO}_{4}$, and 10 HEPES, $\mathrm{pH}$ 7.7. High-divalent ASW contained the following (in mM): $368 \mathrm{NaCl}, 8 \mathrm{KCl}, 13.8 \mathrm{CaCl}_{2}, 100 \mathrm{MgCl}_{2}, 20 \mathrm{MgSO}_{4}$, 10 HEPES, $\mathrm{pH}$ 7.7. The surgical removal of connective sheath tissue (desheathing) from ganglia (both in isolated ganglia and reduced tail preparations) was performed in equal volumes of isotonic $\mathrm{MgCl}_{2}$ and normal ASW. All recordings from isolated ganglia took place in highdivalent ASW, which reduces excitability and blocks the polysynaptic inputs to the motor neuron. All recordings from reduced tail preparations took place in normal ASW to assess the peripheral excitability of tail sensory neurons.

Intact animals. The animal training and testing procedures have been described previously in detail (Wainwright et al., 2002). Briefly, the tailelicited siphon withdrawal reflex was evoked by weak electrical stimulation of the tail. Test stimuli [20 ms alternating current (AC)] were delivered bilaterally through Teflon-coated silver wires implanted $1 \mathrm{~cm}$ rostrally to the tip of the tail and $0.5 \mathrm{~cm}$ laterally to the midline. The intensity of test stimuli was adjusted to $2 \times$ reflex threshold. Training started after the recording of baseline behavior (five test stimuli on each side at 10 min intervals, alternating between the two sides of the tail). A single training session consisted of four trains $(1 \mathrm{~Hz}, 10 \mathrm{~s})$ of sensitizing stimuli $(500 \mathrm{~ms}, 60 \mathrm{~mA} \mathrm{AC})$ repeated at 30 min intervals. Sensitizing stimuli were delivered to the lateral body wall outside the receptive field of tail sensory neurons (see Fig. 1). Four identical training sessions were performed at $24 \mathrm{~h}$ intervals. The post-training behavioral measurement was performed $24 \mathrm{~h}$ after the last day of training, on day 5, and was identical to the pretraining testing session. After each test stimulus, the duration of siphon withdrawal, from the onset of contraction to the onset of relaxation, was measured by a "blind" observer. Averages of the five test scores before and after LTS training were calculated and their ratio was used for between-group comparisons of the training effect. All experiments were conducted at $15^{\circ} \mathrm{C}$. One-sided training of animals for sensitization has been frequently used in the past, because it provides for an additional control within the trained animals (i.e., the contralateral side) (Scholz and Byrne, 1987; Goldsmith and Byrne, 1993; Cleary et al., 1998; Wainwright et al., 2002, 2004; Antzoulatos et al., 2006). In particular, the $4 \mathrm{~d}$ training for LTS has been shown previously to lead to robust electrophysiological and morphological changes in tail sensory neurons (Wainwright et al., 2002, 2004).

Isolated ganglia. Animals were anesthetized by injection of isotonic $\mathrm{MgCl}_{2}(0.5 \mathrm{ml} / \mathrm{g})$. Intact pleural-pedal ganglia were removed from the animal and were pinned to the Sylgard-coated floor of a recording chamber. After the ganglia were desheathed, they were bathed in high-divalent ASW for at least $30 \mathrm{~min}$ before recordings. Recordings were made by a blind observer.

The synaptic stimulation procedures have been described previously in detail (Phares et al., 2003). Briefly, sensory neurons in the ventrocaudal cluster of the pleural ganglion were impaled with a single electrode $(10-15 \mathrm{M} \Omega$ ), whereas postsynaptic motor neurons in the pedal ganglion were impaled with two electrodes: one to record membrane potential and another to inject current. Input resistance of sensory and motor neurons was tested by injecting a $1 \mathrm{~s}$ square pulse of $-1 \mathrm{nA}$ current and measuring the steady-state hyperpolarization. To test synaptic depression, the motor neuron was hyperpolarized to $-80 \mathrm{mV}$ and the sensory neuron was stimulated to fire 10 single spikes at either $100 \mathrm{~ms}$ or $10 \mathrm{~s}$ intervals. Synapses were tested at both frequencies at a $15 \mathrm{~min}$ interval. A single pair of sensory and motor neurons was tested at each preparation. Data were acquired with Clampex 8.2 and analyzed with Clampfit 9.0 (Molecular Devices, Union City, CA).

The peak amplitude of all EPSPs that were evoked at $0.1 \mathrm{~Hz}$ was measured from the immediately preceding baseline, as was the peak amplitude of the initial EPSP $\left(\operatorname{EPSP}_{1}\right)$ at the $10 \mathrm{~Hz}$ train. The amplitude of EPSP $_{2}$ to EPSP 10 that was evoked at $10 \mathrm{~Hz}$ was measured from the extrapolated decay of the preceding EPSP. However, the results of across- group comparisons were identical if the net membrane depolarization at the peak of EPSPs was used, without correction for the decay of the preceding EPSP (data not shown). For both frequencies of stimulation, the average amplitude of $\mathrm{EPSP}_{5}$ to $\mathrm{EPSP}_{10}$ represented the steady state of depression (i.e., EPSP $_{\mathrm{ss}}$ ). Percent depression at steady state was calculated as $100 \times \mathrm{EPSP}_{\text {ss }} / \mathrm{EPSP}_{1}$. In preparations in which there was not sufficient synaptic activation (e.g., because of spike failures), only the first one or two EPSPs were included in the analysis. Previous analyses have suggested that, despite the summation of membrane depolarization in unclamped cells during $10 \mathrm{~Hz}$ stimulation, the relative depression of EPSPs matches that of the depression of EPSCs, even when the synapses are facilitated (Phares and Byrne, 2005).

To quantify the spike width, sensory neurons were impaled with two sharp glass electrodes $(10-15 \mathrm{M} \Omega)$. One electrode was used to inject current, whereas the other was used to record membrane potential. As in synaptic stimulation experiments, single spikes were triggered by injecting $10 \mathrm{~ms}$ square pulses of $2-5 \mathrm{nA}$ depolarizing current. Because we observed that repetitive stimulation of cells in the pleural ganglion could lead to spike broadening and mask the spike narrowing effect of LTS, we only used data from the first sensory neuron tested in each preparation. For the same reason, the majority of data on spike width and synaptic depression $(>95 \%)$ were acquired from separate preparations. Data were acquired with Clampex 8.2 and analyzed with Clampfit 9.0. Spike width was quantified at $50 \%$ of peak amplitude, and decay time was quantified between 100 and 10\% of peak amplitude. All dissections and experiments on isolated ganglia were performed at $15^{\circ} \mathrm{C}$.

Reduced tail preparations. Instead of injecting animals with isotonic $\mathrm{MgCl}_{2}$, which cannot be readily washed out of peripheral tissue and may reduce the peripheral excitability of sensory neurons (our unpublished observations), animals were anesthetized by cooling in ice for $20 \mathrm{~min}$ (Stopfer et al., 1996). The anesthetized animal was then placed on a rectangular dissection tray, dorsal surface up, and was pinned down through the anterior tentacles and the parapodia. The left side of its tail was implanted with a pair of Teflon-coated silver wires, as was the case for animals that underwent behavioral testing (see above).

A longitudinal incision from the mantle cavity to the head was made. All internal organs and central ganglia, except for the left pleural pedal, were removed. The nerves leaving the left pleural-pedal ganglia were severed, except for LP9, which innervates the left posterior tail (i.e., the site where the electrodes were implanted). The reduced preparation was transferred to a recording chamber, pinned down, and bathed in normal ASW. The left pleural-pedal ganglia were pinned down on a Sylgardcoated platform, which was isolated from the rest of the preparation with a narrow Vaseline partition, and contained a medium of ASW and $\mathrm{MgCl}_{2}$. Vaseline application was limited to no more than $\sim 1 \mathrm{~mm}$ of the nerve, as more extensive partitions were observed to block conduction of peripheral spikes. After the pleural ganglion was desheathed, the bathing medium was switched to normal ASW. The preparation was rested for at least $30 \mathrm{~min}$ before the onset of the experiment.

Because we were interested in the responses elicited by the test stimuli used on intact animals, in each preparation we stimulated the tail through the implanted silver-wire electrodes while searching for responsive sensory neurons. To avoid spike-broadening effects of repetitive stimulation, each candidate sensory neuron was tested with a single stimulus to the tail. Unless a responsive sensory neuron was identified within the first 10 tests, the preparation was discarded. In most preparations that were not discarded, $4-5$ tests were sufficient to identify responsive sensory neurons. Test stimuli consisted of 20 and $200 \mathrm{~ms}$ AC shock $(60 \mathrm{~Hz})$ provided by a variable transformer for which the output was gated by a relay. As all test and training stimuli used in our study and other studies (Cleary et al., 1998; Wainwright et al., 2002, 2004; Antzoulatos et al., 2006) were $\mathrm{AC}(60 \mathrm{~Hz})$, the responses of sensory neurons to $60 \mathrm{~Hz}$ stimuli is particularly relevant for the animal's behavioral response. The output of the variable transformer passed through a $20 \mathrm{k} \Omega$ resistor to provide a quasiconstant current. The short circuit current that passed through this circuit for test stimuli was $\sim 5 \mathrm{~mA}$. Recordings were made by a blind observer. The afferent discharge was recorded passively from the soma of the sensory neuron with an intracellular electrode (10-15 M $\Omega$ ). Spike waveform was quantified as described above for isolated ganglia. 


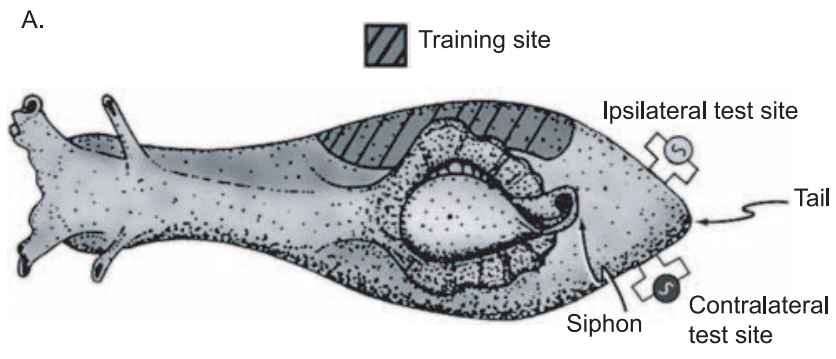

B.
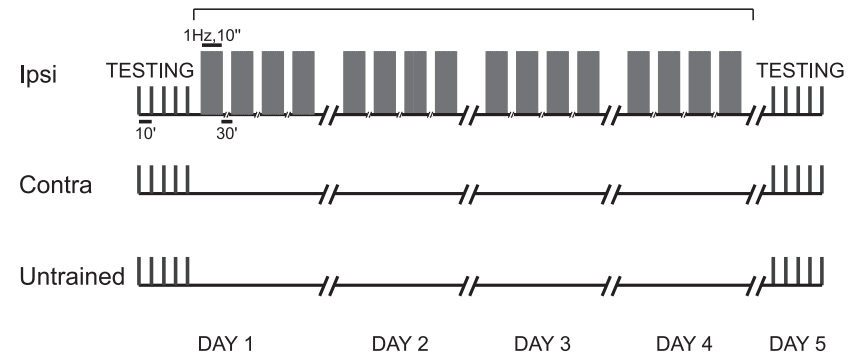

Figure 1. Protocol for sensitization training. A, All training stimuli were delivered on a randomly chosen side of the lateral body wall. Testing stimuli were delivered on the ipsilateral and contralateral sides of the tail, at an area different from the one where training stimuli were delivered. $\boldsymbol{B}$, Four-day training for LTS started immediately after a session of baseline behavioral testing. Twenty-four hours after the end of training, an identical behavioral testing session was used to assess LTS on the ipsilateral side of trained animals versus the contralateral side of trained animals or the untrained animals.

Between-group comparisons on spike waveform were performed only on data from a single sensory neuron per preparation. To analyze the regression between spike width and sensory neuron responsiveness, two sensory neurons were occasionally used from a single preparation. All dissections and recordings in reduced preparation experiments were performed at room temperature $\left(\sim 22^{\circ} \mathrm{C}\right)$ because the large heat load prevented cooling of the entire chamber to $15^{\circ} \mathrm{C}$.

Statistics. Unless otherwise noted, data were analyzed with one-way ANOVA, followed by Student-Newman-Keuls post hoc comparisons. Values are reported as mean \pm SEM. A correlation analysis between behavioral and physiological data were not performed because there were insufficient data from animals that were tested both in vivo and in vitro.

\section{Results}

Prolonged training leads to ipsilateral long-term sensitization Four daily sessions of sensitization training led to a robust increase in the duration of siphon withdrawal, which was elicited by weak stimulation of the ipsilateral side of the tail $24 \mathrm{~h}$ after the end of training (Fig. 1). The average post/pre ratio of response duration was significantly larger for test stimuli delivered to the ipsilateral side of trained animals than for those delivered to the contralateral side of trained animals or to untrained animals (Fig. $2 A$ ) [ipsilateral (Ipsi), $2.17 \pm 0.31, n=18$; contralateral (Contra), $1.15 \pm 0.12, n=17$; Untrained, $1.32 \pm 0.22, n=16 ; F_{(2,48)}=$ 5.69; $p<0.01$; individual post hoc comparisons, Ipsi vs Contra, $q=4.44, p<0.05$; Ipsi vs Untrained, $q=3.65, p<0.05$; Contra vs Untrained, $q=0.71, p=0.61]$. In the subset of trained animals that were tested bilaterally, the responses elicited by tail stimulation on the side ipsilateral to LTS training were significantly longer in duration than the responses elicited by tail stimulation on the contralateral side (Ipsi, $8.93 \mathrm{~s} \pm 1.42$; Contra, $5.12 \mathrm{~s} \pm$ $0.70 ; t_{(16)}=3.17 ; p<0.01, t$ test for paired samples). These data indicate that, as also observed previously (Wainwright et al., 2002, 2004; Antzoulatos et al., 2006), this training procedure en-
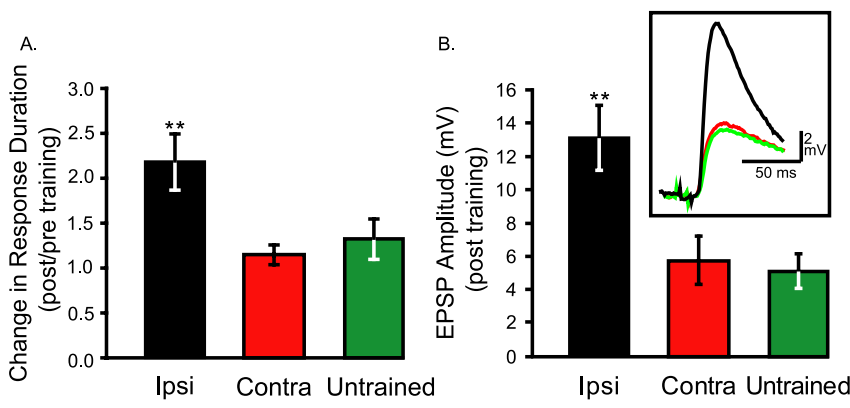

Figure 2. Long-term sensitization training induces parallel effects on behavior of animals and efficacy of sensorimotor synapses. $\boldsymbol{A}$, Average data on the changes in duration of siphonwithdrawal reflex $24 \mathrm{~h}$ after $4 \mathrm{~d}$ LTS training. The tail-elicited siphon withdrawal reflex is sensitized only when the ipsilateral side of the tail is tested. When the contralateral side is tested, the reflex is similar to that elicited by stimulation of untrained animals, indicating that longterm memory for sensitization is unilateral. $\boldsymbol{B}$, Average data on the peak amplitude of monosynaptic EPSPs recorded from the cell body of motor neurons after triggering spikes in presynaptic sensory neurons. In pleural-pedal ganglia of ipsilaterally trained animals, EPSPs had significantly larger amplitude than in synapses from contralaterally trained and untrained animals. Inset, example traces of EPSPs. ${ }^{* *} p<0.01$.

hanced the effectiveness of stimulation of ipsilateral sensory neurons to elicit withdrawal responses. Long-term memory for sensitization is presumably mediated by changes of the ipsilateral tail sensory neurons, as well as other downstream sites of the tailelicited siphon withdrawal network (Trudeau and Castellucci, 1992; Cleary et al., 1995).

Long-term facilitation of sensorimotor synapses preserves the dynamics of postsynaptic responses to presynaptic activity We next examined the sensorimotor synapses in isolated pleuralpedal ganglia from animals that received LTS training to characterize their efficacy and dynamic properties. Ganglia were isolated 22-26 $\mathrm{h}$ after the end of $4 \mathrm{~d}$ LTS training or control treatment and sensorimotor synapses were stimulated with 10 presynaptic action potentials at 10 and $0.1 \mathrm{~Hz}$.

EPSP $_{1}$ of the first train of spikes challenging a synapse represented the level of synaptic efficacy. Synapses were tested at least 15 min after the sensory neuron was tested for spike threshold, as intervals longer than $10 \mathrm{~min}$ are considered sufficient for the synaptic properties to recover from mild previous stimulation (Phares and Byrne, 2005). As reported previously (Frost et al., 1985; Wainwright et al., 2004), synaptic efficacy was elevated in ganglia from animals that had received training on the ipsilateral side of the lateral body wall (Fig. $2 B$ ) (Ipsi, $13.12 \pm 1.92 \mathrm{mV}, n=$ 8; Contra, $5.91 \pm 1.50 \mathrm{mV}, n=6$; Untrained, $5.22 \pm 1.01 \mathrm{mV}$, $n=9 ; F_{(2,20)}=8.78 ; p<0.005$; individual post hoc comparisons. Ipsi vs Contra, $q=4.53, p<0.05$; Ipsi vs Untrained, $q=5.52, p<$ 0.05 ; Contra vs Untrained, $q=0.45, p=0.75)$. The observed long-term facilitation of the sensorimotor synapse was not accompanied by changes in the resting membrane potential and input resistance of sensory or motor neurons, in the decay time, or half-width of EPSPs (Table 1). These results suggest that LTS training, in parallel with the behavioral memory for sensitization, also led to long-term facilitation of the synapses of ipsilateral sensory neurons and, consequently, increased their potential to recruit postsynaptic target cells.

Despite the long-term facilitation of ipsilateral sensorimotor synapses, there was no change in the dynamics of postsynaptic responses to presynaptic activity. The paired-pulse ratio $\left(\mathrm{EPSP}_{2} /\right.$ EPSP $\left._{1} \times 100 \%\right)$ at $100 \mathrm{~ms}$ interpulse interval was not different among the three groups of synapses (Fig. 3A1,A2) (Ipsi, $54.16 \pm$ 
Table 1. Comparison of physiological properties across three groups of animals

\begin{tabular}{|c|c|c|c|c|}
\hline Variable & Ipsi & Contra & Untrained & ANOVA \\
\hline SN Vresting (mV) & $-46.98 \pm 2.77$ & $-44.80 \pm 2.36$ & $-51.10 \pm 3.22$ & $p=0.36$ \\
\hline SN input resistance (M $\Omega$ ) & $28.57 \pm 3.34$ & $19.21 \pm 4.41$ & $30.20 \pm 7.25$ & $p=0.31$ \\
\hline MN Vresting (mV) & $-51.32 \pm 4.99$ & $-48.07 \pm 2.08$ & $-50.25 \pm 3.03$ & $p=0.85$ \\
\hline MN input resistance $(\mathrm{M} \Omega$ ) & $12.33 \pm 2.52$ & $12.05 \pm 2.15$ & $13.93 \pm 2.59$ & $p=0.85$ \\
\hline $\mathrm{EPSP}^{a}$ peak $(\mathrm{mV})$ & $12.71 \pm 1.65$ & $6.14 \pm 1.49$ & $5.59 \pm 1.22$ & $p<0.005$ \\
\hline EPSP width (ms) & $84.01 \pm 12.04$ & $71.95 \pm 9.56$ & $77.54 \pm 12.53$ & $p=0.76$ \\
\hline EPSP decay time (ms) & $269.28 \pm 49.36$ & $273.32 \pm 50.66$ & $189.58 \pm 48.04$ & $p=0.46$ \\
\hline Spike $^{b}$ peak (mV) & $98.54 \pm 2.92$ & $88.38 \pm 2.77$ & $90.16 \pm 3.06$ & $p=0.06$ \\
\hline Spike ${ }^{b}$ width (ms) & $2.92 \pm 0.14$ & $3.46 \pm 0.15$ & $3.54 \pm 0.11$ & $p<0.05$ \\
\hline Spike $e^{b}$ decay time (ms) & $3.96 \pm 0.30$ & $4.10 \pm 0.14$ & $4.55 \pm 0.11$ & $p=0.08$ \\
\hline Spike ${ }^{c}$ peak (mV) & $90.08 \pm 4.17$ & $75.40 \pm 2.93$ & $80.78 \pm 3.15$ & $p<0.05^{d}$ \\
\hline Spike' width (ms) & $1.74 \pm 0.09$ & $2.54 \pm 0.22$ & $2.32 \pm 0.12$ & $p<0.05$ \\
\hline Spike ${ }^{c}$ decay time (ms) & $2.67 \pm 0.23$ & $3.49 \pm 0.24$ & $3.76 \pm 0.48$ & $p=0.14$ \\
\hline Spike' rise time (ms) & $1.33 \pm 0.03$ & $1.87 \pm 0.19$ & $1.58 \pm 0.08$ & $p<0.05^{d}$ \\
\hline
\end{tabular}

Data are represented as mean \pm SEM and significant differences appear in bold. SN, Sensory neuron; MN, motor neuron; Vresting, resting potential. ${ }^{a}$ All EPSP variables were measured from the first EPSP of $0.1 \mathrm{~Hz}$ trains of spikes for which the entire trace was visible. The average EPSP amplitude reported in the text includes values both from 0.1 and $10 \mathrm{~Hz}$ trains and is slightly different from the one reported here.

${ }^{b}$ Spikes recorded from isolated ganglia. Rise time could not be reliably measured because of the stimulus-evoked prepotential.

'Spikes recorded from reduced preparations.

${ }^{d}$ The difference is significant only between ipsilateral and contralateral groups.

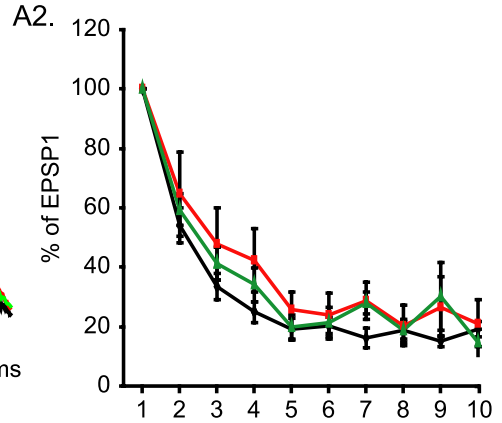

B1.

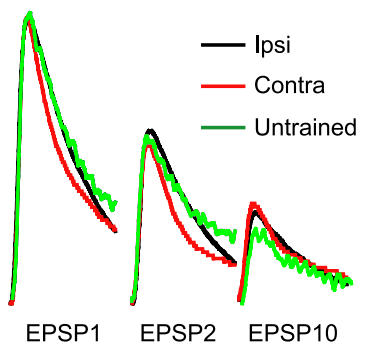

$\overline{100 \mathrm{~ms}}$
B2.

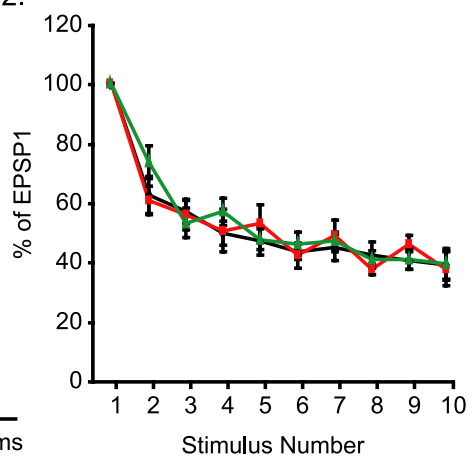

Figure 3. Long-term sensitization does not modify the temporal dynamics of sensorimotor synapses. $\boldsymbol{A} \mathbf{1}$, Example traces of the relative depression of motor neuron responses during $1 \mathrm{~s}$, $10 \mathrm{~Hz}$ stimulation of spikes at presynaptic sensory neurons. These recordings come from animals that had received ipsilateral or contralateral LTS training, or from untrained animals. Traces have been scaled to match in the peak amplitude of the first EPSP. A2, Average data of synaptic depression induced by $10 \mathrm{~Hz}$ stimulation. For each synapse tested, EPSP amplitudes were normalized to the amplitude of the first EPSP. There was no difference among the three groups on the depression achieved with 10 stimuli at $10 \mathrm{~Hz}$. B1, Example traces of the relative depression of motor neuron responses during repeated stimulation at 10 s intervals. The traces (scaled as in A1) come from ipsilaterally trained, contralaterally trained, or untrained animals. $\boldsymbol{B 2}$, Average data of synaptic depression induced by $0.1 \mathrm{~Hz}$ stimulation. For each synapse tested, EPSP amplitudes were normalized to the amplitude of the first EPSP. There was no difference among the three groups on the depression achieved with 10 stimuli at $0.1 \mathrm{~Hz}$.

$5.83 \%, n=6$; Contra, $64.73 \pm 14.16 \%$, $n=6$; Untrained, $59.35 \pm 5.31 \%, n=8$; $\left.F_{(2,17)}=0.33 ; p=0.72\right)$. In addition, after 10 stimuli, all three groups of synapses depressed to a steady state that was $\sim 20 \%$ of EPSP $_{1}$ (Fig. 3A1,A2) (Ipsi, $18.77 \pm 2.52 \%$, $n=6$; Contra, $23.22 \pm 6.36 \%, n=6$; Untrained, $24.62 \pm 5.05 \%, n=7 ; F_{(2,16)}=$ $0.38 ; p=0.69)$.

Similarly, the dynamics of synaptic depression with $0.1 \mathrm{~Hz}$ stimulation were not affected by LTS training. The paired-pulse ratio at $10 \mathrm{~s}$ interpulse interval was not different among the three groups of synapses (Fig. 3B1,B2) (Ipsi, 63.01 $\pm 3.29 \%, n=7$; Contra, $61.25 \pm 4.79 \%, n=5$; Untrained, $74.10 \pm 5.58 \%, n=7 ; F_{(2,16)}=2.26 ; p=$ $0.14)$. When stimulated with 10 spikes at $10 \mathrm{~s}$ intervals, all three groups of synapses depressed to a steady state that was $\sim 40 \%$ of the initial EPSP (Fig. 3B1,B2) (Ipsi, $41.87 \pm 3.00 \%, n=6$; Contra, $42.69 \pm$ $2.80 \%, n=5$; Untrained, $43.27 \pm 1.66 \%, n=7 ; F_{(2,15)}=0.09$; $p=0.92)$. These results collectively suggest that LTS training led to long-term facilitation of ipsilateral sensorimotor synapses, while maintaining the temporal dynamics of postsynaptic responses.

\section{Long-term sensitization involves spike narrowing in sensory neurons}

The effects of LTS training on the waveform of sensory neuron spikes were examined both in isolated pleural-pedal ganglia (Fig. $4 A 1$ ) and in reduced tail preparations (Fig. 4B1). In isolated ganglia, single spikes were triggered by injecting positive current in the cell body of the sensory neuron through one electrode while the resultant spike was recorded through a second electrode. LTS significantly affected the waveform of ipsilateral sensory neuron spikes, primarily by reducing their width (Fig. $4 A 2, A 3$ ) (Ipsi, $2.92 \pm 0.14 \mathrm{~ms}, n=7$; Contra, $3.46 \pm 0.15 \mathrm{~ms}, n=$ 9; Untrained, $3.54 \pm 0.11 \mathrm{~ms}, n=9 ; F_{(2,22)}=5.67 ; p<0.05$; individual post hoc comparisons, Ipsi vs Contra, $q=3.88, p<$ 0.05 ; Ipsi vs Untrained, $q=4.47, p<0.05$; Contra vs Untrained, $q=0.63, p=0.67)$. There were no differences among the three groups on other properties of spikes (i.e., amplitude and decay time) (data not shown).

A similar effect of LTS training on spike width was apparent in reduced tail preparations, in which spikes were triggered through peripheral AC stimulation of the tail and recorded with a single electrode in the cell body. LTS training significantly reduced the width of ipsilateral sensory neuron spikes (Fig. 4B2,B3, Table 1) (Ipsi, $1.74 \pm 0.09 \mathrm{~ms}, n=5$; Contra, $2.54 \pm 0.22 \mathrm{~ms}, n=7$; Untrained, $2.32 \pm 0.12 \mathrm{~ms}, n=7 ; F_{(2,16)}=5.63 ; p<0.05$; individual post hoc comparisons, Ipsi vs Contra, $q=4.68, p<$ 0.05 ; Ipsi vs Untrained, $q=3.37, p<0.05$; Contra vs Untrained, $q=1.43, p=0.90)$. As was the case for isolated ganglia, there were no differences in decay time. In contrast to the results from isolated ganglia, the spikes recorded in reduced preparations displayed a difference in peak amplitude and rise time, which was only apparent between the ipsilateral and contralateral groups (Table 1). These results demonstrate that, after LTS training, the spikes of ipsilateral tail sensory neurons, whether triggered at the cell body or peripherally, had an altered waveform, which was expressed most prominently in the spike width. 
Long-term sensitization training enhances the responses of sensory neurons to peripheral stimuli

To examine the peripheral excitability of tail sensory neurons, weak test stimuli were delivered to the skin of the tail while the afferent discharge of sensory neurons was recorded at the cell body. Tail stimulation was similar to what is generally used to elicit weak withdrawal responses (Scholz and Byrne, 1987; Goldsmith and Byrne, 1993; Cleary et al., 1998; Sutton et al., 2001; Wainwright et al., 2002, 2004; Antzoulatos et al., 2006). As also reported previously (Phares et al., 2003), the spiking responses to cutaneous $\mathrm{AC}(60 \mathrm{~Hz})$ stimuli had short latency, were time-locked to the AC stimulus, and included high-frequency firing (approaching $60 \mathrm{~Hz}$ ), sometimes followed by a highly variable afterdischarge. The initial response to a $5 \mathrm{~mA}, 20$ ms AC stimulus ( 1.2 cycles) included either single or double spikes. Given the brief duration of the stimulus (20 ms), we hypothesized that responsive sensory neurons would fire single, as opposed to double spikes. Indeed, in the majority of animals that had received contralateral LTS training, the sensory neurons fired single spikes (six of seven; i.e., spike doublets were only present in $14 \%$ of preparations) (Fig. 5A). A similar result was obtained in the group of animals that had received control treatment (five of six; i.e., spike doublets were only present in $17 \%$ of preparations) (Fig. 5A). In contrast, in the group of animals that had received ipsilateral LTS, the percentage of single spikes (two of five animals; i.e., spike doublets were present in $60 \%$ of preparations) (Fig. $5 \mathrm{~A}$ ) was significantly different from the expected one (expected percentage, $100 \%$; observed percentage, $40 \% ; z=-2.24 ; p<0.05$; $z$ test for difference of percentage from expected value). This result suggests that, in most animals, LTS training doubled the stimulus-elicited spike input of the withdrawal network (i.e., transformed the typical single-spike response to a spike doublet).

The effects of LTS training on the peripheral excitability of ipsilateral tail sensory neurons were also apparent when a longer test stimulus was used. A 5 mA, $200 \mathrm{~ms}$ AC stimulus ( 12 cycles) triggered at least 12 spikes in $100 \%$ (five of five) of ipsilaterally trained sensory neurons (i.e., no spike failures), as would be expected from the optimal response to an AC stimulus (Fig. $5 B 1, B 2)$. In contrast, the same AC stimulus triggered $<12$ spikes (i.e., suboptimal responses) in 50\% (three of six) of contralaterally trained sensory neurons $(z=2.19 ; p<0.05)$ and in $50 \%$ (three of six) of untrained sensory neurons (expected percentage, $100 \%$; observed percentage, $50 \% ; z=2.19 ; p<0.05$; $z$ test for difference of percentage from expected value). These results are in agreement with the ones reported above. Ipsilateral LTS training improved the peripheral responses of sensory neurons to AC stimuli.

The observed reduction in spike failures during afferent discharges of sensory neurons could be related to the degree of spike
A2.

A3.
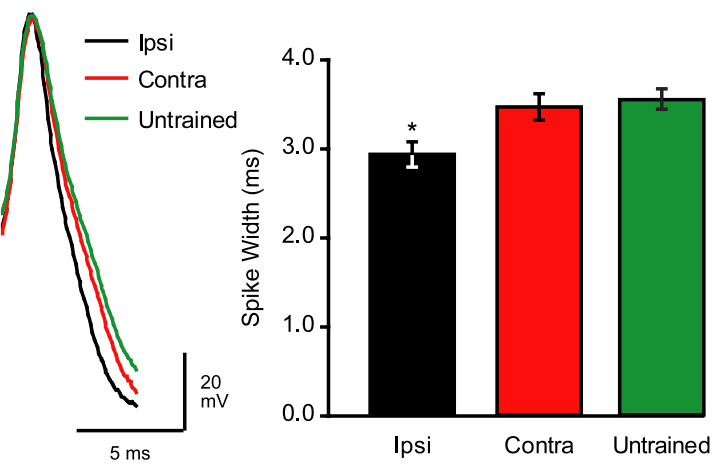

B2.

B3.
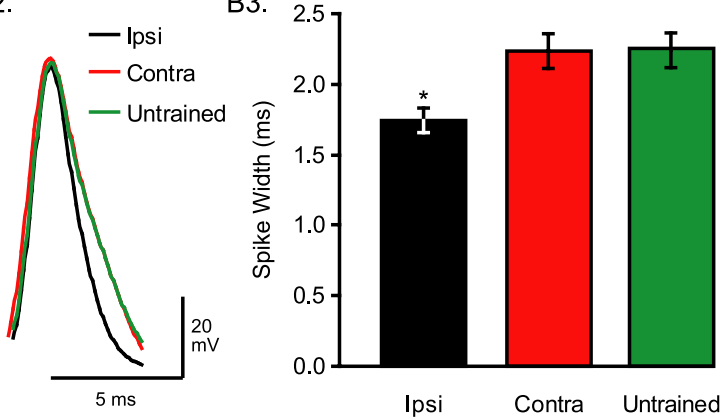

Figure 4. Long-term sensitization is associated with spike narrowing in ipsilateral sensory neurons. $\boldsymbol{A} \mathbf{1}$, In isolated ganglia

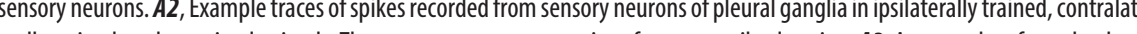
Spikes were narrower in preparations from ipsilaterally trained animals. B3, Average data from the three groups of preparations. Spike width was significantly reduced in ipsilaterally trained animals compared with contralaterally trained and untrained animals. The difference in absolute spike widths between panels $\boldsymbol{A} \mathbf{3}$ and $\mathbf{B} 3$ is caused by the $\sim 7^{\circ}$ difference in temperature. ${ }^{*} p<0.05$.

narrowing. Linear regression analysis of spike data pooled from all cells in all three groups of animals examined whether the number of spikes in the sensory neuron response to the $200 \mathrm{~ms}$ test could be significantly fit by a linear function of spike width. This analysis revealed that spike width, as an independent variable, was a statistically significant regressor (i.e., predictor) of the dependent variable, the $200 \mathrm{~ms}$ evoked spike activity (Fig. 5B3) (slope, $-2.92 ; F_{(1,17)}=10.96 ; p<0.005 ; R^{2}=0.36$ ).

\section{Discussion}

We found that prolonged training for sensitization led to robust long-term behavioral memory, paralleled by robust long-term synaptic facilitation. Long-term sensitization also involved a reduction of the width of centrally and peripherally triggered spikes of tail sensory neurons. To our knowledge, this is the first report of persistent spike narrowing after treatments that induce longterm plasticity. Although LTS was accompanied by spike narrowing and synaptic facilitation, the dynamics of postsynaptic responses to presynaptic activity were preserved. In addition, LTS was accompanied by a reduction in spike failures during afferent discharges of sensory neurons. Because LTS training was performed unilaterally, all effects were expressed on the ipsilateral side only.

Short-term memory for sensitization of Aplysia withdrawal 
A1. Responses to $20 \mathrm{~ms} \mathrm{AC}$

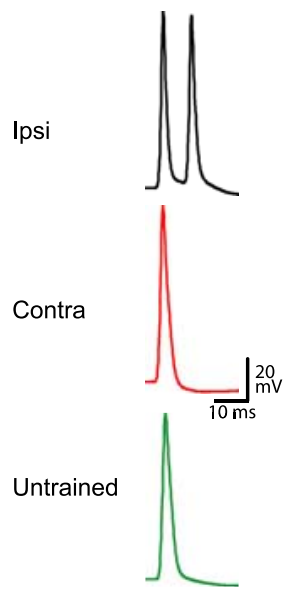

B1. Responses to $200 \mathrm{~ms} \mathrm{AC}$

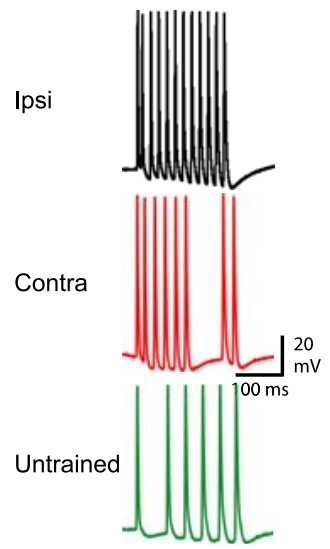

A2.

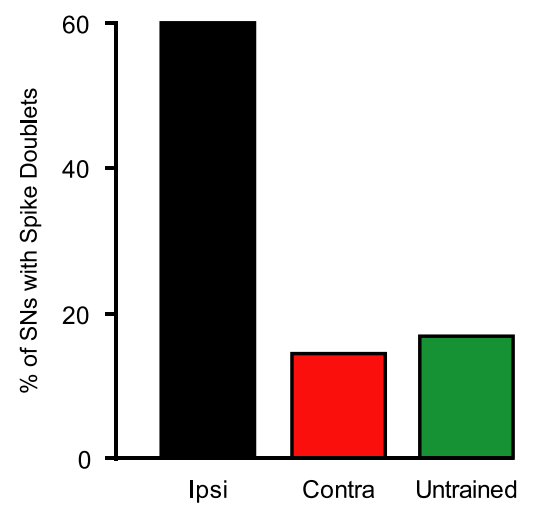

B3.
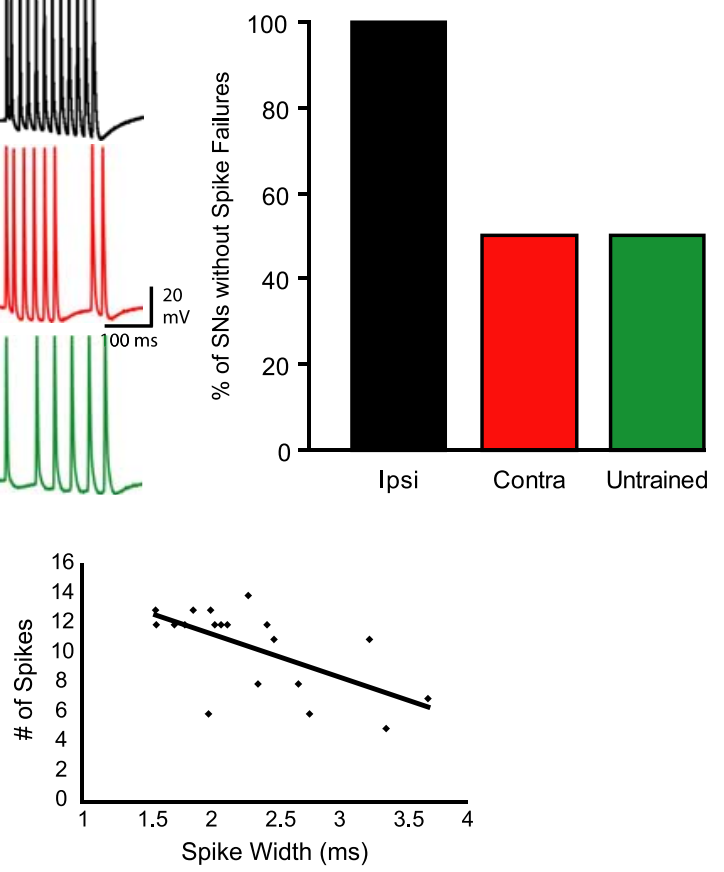

Figure 5. Long-term sensitization enhances the sensory neuron responses to cutaneous stimuli. $\boldsymbol{A} 1$, Example traces of sensory neuron spike activity in an ipsilaterally trained, a contralaterally trained, and an untrained animal in response to a $20 \mathrm{~ms} \mathrm{AC} \mathrm{stimulus} \mathrm{(i.e.,} 1.2$ cycles at $60 \mathrm{~Hz}$. $\mathbf{A 2}, \ln 60 \%$ of ipsilaterally trained preparations, the $20 \mathrm{~ms} \mathrm{AC}$ triggered spike doublets, versus only 14 and $17 \%$ of contralaterally trained and untrained preparations, respectively. The complementary percentages correspond to neurons that fired single spikes. B1, Example traces of sensory neuron spike activity in an ipsilateral, a contralateral, and an untrained preparation, in response to a $200 \mathrm{~ms} \mathrm{AC}$ stimulus (i.e., 12 cycles at $60 \mathrm{~Hz}$ ). A sensory neuron response included a spike failure if there were $<12$ spikes in the time-locked phase of the response. $B \mathbf{2}$, In $100 \%$ of preparations from ipsilaterally trained animals, sensory neurons fired at least 12 spikes (i.e., no failures) (mean number \pm SEM, $12.60 \pm 0.25$ ). In contrast, in $50 \%$ of preparations from contralateral and untrained animals, sensory neuron responses had at least one spike failure (contra, $10.50 \pm 1.29$; untrained, $9.50 \pm 1.20$ ). $\mathbf{B 3}$, Spike width was a significant predictor of the number of spikes sensory neurons would fire in response to a $200 \mathrm{~ms}$ peripheral stimulus. The line of best fit had a slope of -2.92 .

reflexes is associated with broadening of sensory neuron spikes, an effect mediated by phosphorylation of voltage-gated $\mathrm{K}^{+}$channels (Klein and Kandel, 1980; Klein et al., 1982; Baxter and Byrne, 1989; Byrne and Kandel, 1996; Baxter et al., 1999). Spike broad-

ening is believed to increase the calcium influx in presynaptic terminals, which, in turn, increases the release of glutamate from sensory neurons. Facilitation of the synapses of sensory neurons, in turn, would increase not only the input of motor neurons, but also the input of interneurons. Some of these interneurons mediate feedforward excitation in the withdrawal circuit (Cleary et al., 1995). Computational studies have suggested that, whereas facilitation of the sensorimotor connections would increase the magnitude of motor neuron responses to peripheral stimuli, upregulation of the interneuron activity would increase the duration of the motor neuron responses (White et al., 1993). Indeed, facilitation of sensorimotor synapses has been correlated with increased behavioral responses in reduced preparations (Walters et al., 1983).

Similar to short-term memory, long-term memory in Aplysia has been attributed to facilitation of the synapses of sensory neurons (Frost et al., 1985; Cleary et al., 1998; Wainwright et al., 2004). Surprisingly, changes in spike width produced by LTS have never been examined. The present study suggests that, whereas short-term sensitization involves spike broadening, long-term sensitization involves spike narrowing. Although the mechanism of spike narrowing was not examined here, it would likely involve the upregulation of $\mathrm{K}^{+}$currents (i.e., the opposite mechanism of spike broadening), as also recently demonstrated in Tritonia diomedea (Sakurai et al., 2006). Because the passive electrical properties of the sensory neurons at rest were not affected by LTS training (Table 1), spike narrowing is most likely not mediated by modulation of $\mathrm{K}^{+}$conductances that contribute to the leak current. If spike broadening produces synaptic facilitation through an increase in release probability, how does spike narrowing contribute to memory?

One potential effect of spike narrowing could be a reduction of calcium entry and a consequent decrease in transmitter release (Holz et al., 1986; Mackey et al., 1987; Rosen et al., 1989; Niven and Burrows, 2003) (i.e., the opposite effects of spike broadening). However, spike narrowing did not appear to affect the properties of the sensorimotor synapses. First, long-term sensitization produced synaptic facilitation, not synaptic depression (Fig. 2 B). Second, LTS did not modify the dynamics of activity-dependent depression, as would be expected if there was a change in release probability (Gingrich and Byrne, 1985). A decrease in the fractional depletion of readily releasable transmitter would reduce the rates of depression at least at the $10 \mathrm{~Hz}$ stimulation. In addition, a decrease in transmitter release would reduce desensitization of postsynaptic receptors, and, therefore, the depression evoked by $10 \mathrm{~Hz}$ stimulation (Antzoulatos et al., 2003). One potential explanation is that the modest spike narrowing we found after LTS would not reduce calcium influx sufficiently to change release probability.

Despite the lack of LTS effect on temporal dynamics of synaptic depression, our data cannot conclusively exclude the possibility that there are changes in probability of transmitter release from presynaptic terminals. Such changes may have been masked by nonlinear summation of membrane depolarization (because the motor neurons were not voltage-clamped during recording), or by opposite changes in frequency facilitation that could counteract changes in depression. However, we believe that the masking of changes in transmitter release is unlikely. First, voltageclamping the motor neurons has been shown previously to lead to very similar estimates of presynaptic depression as currentclamping (Phares and Byrne, 2005). Second, changes in homosynaptic facilitation would mostly affect, if at all, the initial 2-3 EPSPs at high frequency (Jiang and Abrams, 1998). LTS failed to 
affect synaptic depression not only on the $10 \mathrm{~Hz}$ paired-pulse ratio, but also on any other aspect of synaptic depression induced by high- and low-frequency stimulation. In addition, whether spike narrowing somewhat changes presynaptic release probability or not, our data suggest that this effect does not contribute to the network output. If it did, the dynamics of postsynaptic responses to high-frequency stimulation should be affected. No change in temporal dynamics of depression was apparent in the data, suggesting that spike narrowing does not affect the dynamics of burst transmission, and therefore, the output of the network.

The preservation of synaptic dynamics after facilitation is consistent with previous reports in Aplysia and the mammalian hippocampus. Short-term heterosynaptic facilitation of sensorimotor synapses does not affect the dynamics of homosynaptic depression at $10 \mathrm{~Hz}$ stimulation (Phares and Byrne, 2005). Longterm habituation does not affect the rate of synaptic depression with frequent stimulation (Castellucci et al., 1978). In addition, long-term facilitation of sensorimotor synapses in vitro, has not been associated with changes in dynamics of activity-dependent depression (Montarolo et al., 1986). Finally, long-term potentiation of hippocampal synapses preserves the fidelity of synaptic transmission (Pananceau et al., 1998; Buonomano, 1999; Selig et al., 1999).

If long-term facilitation does not modify synaptic dynamics, it is most likely mediated by an increase in number of functional synaptic contacts. Any change in release probability would be expected to impinge on the rate of activity-dependent homosynaptic depression, which does not appear to occur in Aplysia (Fig. 3 ) or in any of the synapses mentioned above. The most parsimonious account for long-term synaptic facilitation in the sensorimotor synapse has to rely, therefore, predominantly on the ultrastructural changes that have been associated with long-term facilitation previously (Wainwright et al., 2004). An increase in the number of release sites would increase the postsynaptic response without affecting the synaptic dynamics.

Although the LTS-induced spike narrowing did not modify the dynamics of synaptic transmission, it was associated with an enhancement of the afferent responses of sensory neurons. Sensory neurons with narrow spikes tended to respond to peripheral AC stimuli with fewer spike failures than sensory neurons with normal spike widths, presumably because narrower spikes reduced the refractory period. Therefore, the reduction of spike failures in ipsilateral sensory neurons after LTS may be secondary to the spike narrowing effect of LTS on these neurons. However, the causal relationship between the mechanisms underlying spike narrowing and enhanced excitability needs to be established.

The correlation between spike width and levels of burst activity in Aplysia sensory neurons is consistent with other reports in invertebrate and vertebrate systems. During swim acceleration of the mollusc Clione limacina, spike narrowing is necessary for an increase in the firing rate of interneurons and the consequent increase in the cycle frequency of the central pattern generator (Satterlie et al., 2000). Neurons of the lateral magnocellular nucleus of anterior neostriatum of songbirds undergo developmental modifications that broaden their spike width and, in parallel, reduce their capacity to fire bursts of spikes (Bottjer et al., 1998; Livingston and Mooney, 2001). Similarly, computational and experimental studies suggest that the narrow spike that characterizes the GABAergic fast-spiking neurons of the mammalian brain reduces their refractory period and permits their high spiking rate (Connors and Gutnick, 1990; Rudy and McBain, 2001; GonzalezBurgos et al., 2005; Chen et al., 2006; Povysheva et al., 2006).
Another potential ramification of spike narrowing would be an increase in the dynamic range of spike broadening and the consequent short-term facilitation. In that case, long-term sensitization training would be expected to enhance the expression of short-term sensitization. It would be interesting to examine to what extent the LTS-induced spike narrowing that is reported here contributes to the effect of LTS on additional learning.

\section{References}

Angers A, Fioravante D, Chin J, Cleary LJ, Bean AJ, Byrne JH (2002) Serotonin stimulates phosphorylation of Aplysia synapsin and alters its subcellular distribution in sensory neurons. J Neurosci 22:5412-5422.

Antzoulatos EG, Byrne JH (2004) Learning insights transmitted by glutamate. Trends Neurosci 27:555-560.

Antzoulatos EG, Cleary LJ, Eskin A, Baxter DA, Byrne JH (2003) Desensitization of postsynaptic glutamate receptors contributes to high-frequency homosynaptic depression of Aplysia sensorimotor connections. Learn Mem 10:309-313.

Antzoulatos EG, Wainwright ML, Cleary LJ, Byrne JH (2006) Long-term sensitization training primes Aplysia for further learning. Learn Mem 13:422-425.

Baxter DA, Byrne JH (1989) Serotonergic modulation of two potassium currents in the pleural sensory neurons of Aplysia. J Neurophysiol 62:665-679.

Baxter DA, Canavier CC, Clark Jr JW, Byrne JH (1999) Computational model of the serotonergic modulation of sensory neurons in Aplysia. J Neurophysiol 82:2914-2935.

Bottjer SW, Brady JD, Walsh JP (1998) Intrinsic and synaptic properties of neurons in the vocal-control nucleus IMAN from in vitro slice preparations of juvenile and adult zebra finches. J Neurobiol 37:642-658.

Buonomano DV (1999) Distinct functional types of associative long-term potentiation in neocortical and hippocampal pyramidal neurons. J Neurosci 19:6748-6754.

Byrne JH, Kandel ER (1996) Presynaptic facilitation revisited: state and time dependence. J Neurosci 16:425-435.

Carew TJ, Castellucci VF, Kandel ER (1971) An analysis of dishabituation and sensitization of the gill-withdrawal reflex in Aplysia. Int J Neurosci 2:79-98.

Castellucci V, Kandel ER (1976) Presynaptic facilitation as a mechanism for behavioral sensitization in Aplysia. Science 194:1176-1178.

Castellucci VF, Carew TJ, Kandel ER (1978) Cellular analysis of long-term habituation of the gill-withdrawal reflex of Aplysia californica. Science 202:1306-1308.

Chen N, Chen S, Wu Y, Wang J (2006) The refractory periods and threshold potentials of sequential spikes measured by whole-cell recording. Biochem Biophys Res Commun 340:151-157.

Cleary LJ, Byrne JH, Frost WN (1995) Role of interneurons in defensive withdrawal reflexes in Aplysia. Learn Mem 2:133-151.

Cleary LJ, Lee WL, Byrne JH (1998) Cellular correlates of long-term sensitization in Aplysia. J Neurosci 18:5988-5998.

Connors BW, Gutnick MJ (1990) Intrinsic firing patterns of diverse neocortical neurons. Trends Neurosci 13:99-104.

Dale N, Schacher S, Kandel ER (1988) Long-term facilitation in Aplysia involves increase in transmitter release. Science 239:282-285.

Frost WN, Castellucci VF, Hawkins RD, Kandel ER (1985) Mono-synaptic connections made by the sensory neurons of the gill-withdrawal and siphon-withdrawal reflex in Aplysia participate in the storage of longterm memory for sensitization. Proc Natl Acad Sci USA 82:8266-8269.

Gingrich KJ, Byrne JH (1985) Simulation of synaptic depression, posttetanic potentiation, and presynaptic facilitation of synaptic potentials from sensory neurons mediating gill-withdrawal reflex in Aplysia. J Neurophysiol 53:652-669.

Goldsmith JR, Byrne JH (1993) Bag cell extract inhibits tail-siphon withdrawal reflex, suppresses long-term but not short-term sensitization, and attenuates sensory-to-motor neuron synapses in Aplysia. J Neurosci 13:1688-1700.

Gonzalez-Burgos G, Krimer LS, Povysheva NV, Barrionuevo G, Lewis DA (2005) Functional properties of fast spiking interneurons and their synaptic connections with pyramidal cells in primate dorsolateral prefrontal cortex. J Neurophysiol 93:942-953.

Holz IV GG, Shefner SA, Anderson EG (1986) Serotonin decreases the du- 
ration of action potentials recorded from tetraethylammonium-treated bullfrog dorsal root ganglion cells. J Neurosci 6:620-626.

Jiang XY, Abrams TW (1998) Use-dependent decline of paired-pulse facilitation at Aplysia sensory neuron synapses suggests a distinct vesicle pool or release mechanism. J Neurosci 18:10310-10319.

Klein M, Kandel ER (1980) Mechanism of calcium current modulation underlying presynaptic facilitation and behavioral sensitization in Aplysia. Proc Natl Acad Sci USA 77:6912-6916.

Klein M, Camardo J, Kandel ER (1982) Serotonin modulates a specific potassium current in the sensory neurons that show presynaptic facilitation in Aplysia. Proc Natl Acad Sci USA 79:5713-5717.

Livingston FS, Mooney R (2001) Androgens and isolation from adult tutors differentially affect the development of songbird neurons critical to vocal plasticity. J Neurophysiol 85:34-42.

Mackey SL, Glanzman DL, Small SA, Dyke AM, Kandel ER, Hawkins RD (1987) Tail shock produces inhibition as well as sensitization of the siphon-withdrawal reflex of Aplysia: possible behavioral role for presynaptic inhibition mediated by the peptide Phe-Met-Arg-Phe-NH2. Proc Natl Acad Sci USA 84:8730-8734.

Montarolo PG, Goelet P, Castellucci VF, Morgan J, Kandel ER, Schacher S (1986) A critical period for macromolecular synthesis in long-term heterosynaptic facilitation in Aplysia. Science 234:1249-1254.

Niven JE, Burrows M (2003) Spike width reduction modifies the dynamics of short-term depression at a central synapse in the locust. J Neurosci 23:7461-7469.

Pananceau M, Chen H, Gustafsson B (1998) Short-term facilitation evoked during brief afferent tetani is not altered by long-term potentiation in the guinea-pig hippocampal CA1 region. J Physiol 508:503-514.

Phares GA, Byrne JH (2005) Analysis of 5-HT-induced short-term facilitation at Aplysia sensorimotor synapse during bursts: increased synaptic gain that does not require ERK activation. J Neurophysiol 94:871-877.

Phares GA, Antzoulatos EG, Baxter DA, Byrne JH (2003) Burst-induced synaptic depression and its modulation contribute to information transfer at Aplysia sensorimotor synapses: empirical and computational analyses. J Neurosci 23:8392-8401.

Pinsker HM, Hening WA, Carew TJ, Kandel ER (1973) Long-term sensitization of a defensive withdrawal reflex in Aplysia. Science 182:1039-1042.

Povysheva NV, Gonzalez-Burgos G, Zaitsev AV, Kroner S, Barrionuevo G, Lewis DA, Krimer LS (2006) Properties of excitatory synaptic responses in fast-spiking interneurons and pyramidal cells from monkey and rat prefrontal cortex. Cereb Cortex 16:541-552.
Rosen SC, Susswein AJ, Cropper EC, Weiss KR, Kupfermann I (1989) Selective modulation of spike duration by serotonin and the neuropeptides, FMRFamide, SCPB, buccalin and myomodulin in different classes of mechanoafferent neurons in the cerebral ganglion of Aplysia. J Neurosci 9:390-402.

Rudy B, McBain CJ (2001) Kv3 channels: voltage-gated $\mathrm{K}^{+}$channels designed for high-frequency repetitive firing. Trends Neurosci 24:517-526.

Sakurai A, Darghouth NR, Butera RJ, Katz PS (2006) Serotonergic enhancement of a 4-AP-sensitive current mediates the synaptic depression phase of spike timing-dependent neuromodulation. J Neurosci 26:2010-2021.

Satterlie RA, Norekian TP, Pirtle TJ (2000) Serotonin-induced spike narrowing in a locomotor pattern generator permits increases in cycle frequency during accelerations. J Neurophysiol 83:2163-2170.

Scholz KP, Byrne JH (1987) Long-term sensitization in Aplysia: biophysical correlates in tail sensory neurons. Science 235:685-687.

Selig DK, Nicoll RA, Malenka RC (1999) Hippocampal long-term potentiation preserves the fidelity of postsynaptic responses to presynaptic bursts. J Neurosci 19:1236-1246.

Stopfer M, Chen X, Tai YT, Huang GS, Carew TJ (1996) Site specificity of short-term and long-term habituation in the tail-elicited siphon withdrawal reflex of Aplysia. J Neurosci 16:4923-4932.

Sutton MA, Masters SE, Bagnall MW, Carew TJ (2001) Molecular mechanisms underlying a unique intermediate phase of memory in Aplysia. Neuron 31:143-154.

Trudeau LE, Castellucci VF (1992) Contribution of polysynaptic pathways in the mediation and plasticity of Aplysia gill and siphon withdrawal reflex: evidence for differential modulation. J Neurosci 12:3838-3848.

Wainwright ML, Zhang H, Byrne JH, Cleary LJ (2002) Localized neuronal outgrowth induced by long-term sensitization training in Aplysia. J Neurosci 22:4132-4141.

Wainwright ML, Byrne JH, Cleary LJ (2004) Dissociation of morphological and physiological changes associated with long-term memory in Aplysia. J Neurophysiol 92:2628-2632.

Walters ET, Byrne JH, Carew TJ, Kandel ER (1983) Mechanoafferent neurons innervating tail of Aplysia. II. Modulation by sensitizing stimulation. J Neurophysiol 50:1543-1559.

White JA, Ziv I, Cleary LJ, Baxter DA, Byrne JH (1993) The role of interneurons in controlling the tail-withdrawal reflex in Aplysia: a network model. J Neurophysiol 70:1777-1786. 